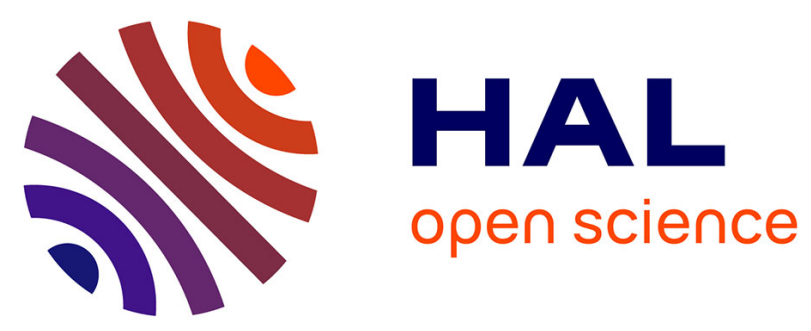

\title{
Determination of the elastic moduli and residual stresses of freestanding Au-TiW bilayer thin films by nanoindentation
}

Matteo Ghidelli, Marco Sebastiani, Christian Collet, Raphael Guillemet

\section{- To cite this version:}

Matteo Ghidelli, Marco Sebastiani, Christian Collet, Raphael Guillemet. Determination of the elastic moduli and residual stresses of freestanding $\mathrm{Au}$-TiW bilayer thin films by nanoindentation. Materials \& Design, 2016, 106, pp.436-445. 10.1016/j.matdes.2016.06.003 . hal-03255146

\author{
HAL Id: hal-03255146 \\ https://hal.science/hal-03255146
}

Submitted on 9 Jun 2021

HAL is a multi-disciplinary open access archive for the deposit and dissemination of scientific research documents, whether they are published or not. The documents may come from teaching and research institutions in France or abroad, or from public or private research centers.
L'archive ouverte pluridisciplinaire HAL, est destinée au dépôt et à la diffusion de documents scientifiques de niveau recherche, publiés ou non, émanant des établissements d'enseignement et de recherche français ou étrangers, des laboratoires publics ou privés. 


\title{
Determination of the elastic moduli and residual stresses of freestanding Au-TiW bilayer thin films by nanoindentation
}

\author{
Matteo Ghidelli ${ }^{1}$, Marco Sebastiani ${ }^{1}$, Christian Collet $^{2}$, Raphael Guillemet ${ }^{2}$ \\ 1،Roma Tre University, Engineering Department, Via della Vasca Navale 79, 00146 Rome, Italy \\ ${ }^{2}$ THALES Research \& Technology, 1 avenue Augustin Fresnel, 91767 Palaiseau, France \\ e-mail: matteo.ghidelli@uniroma3.it,marco.sebastiani@uniroma3.it
}

\begin{abstract}
In this paper, we present a detailed mechanical characterization of freestanding bilayer (Au-TiW) micro-cantilevers and double clamped beams, for applications as Radio Frequency (RF)-switches Micro-Electromechanical Systems (MEMS). The testing structures have been characterized by an optical profilometer and Scanning Electron Microscopy (SEM) equipped with Energy Dispersive X-ray Spectroscopy (EDS), in order to acquire information about their geometries, composition, and the gap between the substrate underneath. Then, the micro-beams are deflected by using a specifically designed nanoindentation procedure based dynamic stiffness measurement during bending in order to extract the elastic modulus and the residual stresses of both layers. Firstly, the classic beam theory has been implemented for bilayer cantilevers enabling the extraction of elastic moduli. Then, residual stresses are estimated by deflecting double clamped beams, while implementing new analytical models for a bilayer system. The obtained elastic moduli are consistent with the average ones obtained for a single layer micro-cantilever and with nanoindentation results for $\mathrm{TiW}$ and $\mathrm{Au}$ homogeneous films. The
\end{abstract}


residual stresses are in agreement with the values obtained from the double slot Focused Ion Beam (FIB) and Digital Image Correlation (DIC) procedure, providing an alternative and portable way for the assessment of residual stresses on composite double clamped micro-beams.

Keywords: Micro Electro-Mechanical Systems; Elastic modulus, Residual stress; Nanoindentation; Focused Ion Beam. 


\section{Introduction}

Micro Electro-Mechanical Systems (MEMS) have found a growing number of applications involving sensors, actuators to active Radio Frequency (RF) components, optics as well as energy generation devices [1]. The assessment of reliability and life time of MEMS devices is a major concern in the microelectronic industry, requiring a full understanding of the mechanical properties of micrometer-sized materials employed in MEMS fabrication [1]. Among other factors, residual stress has been identified as one of the main parameters affecting both performance and lifetime of doubleclamped MEMS structures [1].

Small-scale dimension materials exhibit mechanical properties which are different from their bulk counterpart because of the dominance of surface effects possible microstructural changes [2]. In crystalline materials, the thickness confinement prevents dislocation motion [3] and limits the grain growth favoring strengthening phenomena [2]. The nucleation and propagation of shear bands are also affected by size effects in metallic glasses involving an increase of ductility, yield strength and change in fracture mode $[2,4]$. However, a conclusive understanding of mechanical properties and of the associated deformation mechanisms of small-scale materials is still missing. The difficulty arises from the challenge to generate consistent and reproducible data on small-scale specimens, while avoiding artifacts. Mechanical size effects can affect the mechanisms behind the deformation and fracture of thin films, which can have significant impact on MEMS functionality. Therefore, specific testing methodology are needed to characterize the mechanical properties of thin films.

Many techniques have been employed to investigate freestanding MEMS materials' mechanical behavior including micro-tensile [5], bending [6], bulge tests [7] as well as nano-mechanical testing actuated by residual stress films [8]. Although these techniques can provide useful information about mechanical properties of freestanding films, they often relay upon complicated experimental apparatus which require several steps [6-8] and can only assess specimens with dimensions above few 
micrometers in length and width and $500 \mathrm{~nm}$ in thickness direction [5]. Nanoindentation provides high resolution in load, displacement and $x-y$ positioning and is therefore suitable for characterizing the mechanical response of freestanding thin films [9, 10]. Bending of freestanding cantilever was originally performed by Weihs et al. [11] on Al thin films and has since been applied to other materials such as Ni [12], Ti [13] and metallic glasses [14, 15]. This technique involves the deflection of a freestanding cantilever using a nanoindenter. The stiffness can be extracted as the slope of the loaddeflection curve enabling the determination of the material elastic modulus which is a key parameter in the behavior of MEMS devices [11]. Since then, Guo et al. [14] have minimized the error caused by uncertainties in the effective indentation position performing multiple indentations, Tsou et al. [12] have improved the analysis by subtracting the indentation effect during deflection, while Florando and Nix [16] have used triangular cantilever in order to avoid inhomogeneous strain distribution during bending. Bilayer cantilevers have been tested by Boyd et al. [17] and Fang [18] to extract the elastic moduli using nanoindentation and resonant methods. However, in both cases a complicated Finite Element Modeling (FEM) was required.

Residual stresses are usually extracted measuring the change of substrate curvature consecutive to film deposition using the Stoney Equation [19]. Other techniques based on Focused Ion Beams (FIB) milling can be been employed to precisely account for the effect of the local microstructure [20]. Freestanding MEMS structures can be used to capture the magnitude of the residual stress. Double clamped beams with different length can be used as a strain sensor [21]. Once released, compressive stress can induce buckling above a critical length [21]. An array of beams with different lengths enables to capture the magnitude compressive stress. Tensile residual stress can be extracted using a similar structures involving rings fixed to the substrate at two points with a central beam spanning perpendicularly to the anchor points [22]. After releasing, the tensile internal stress puts the central beam in compression, involving buckling above a critical length [22]. Angular variation of residual 
stresses (both in compression and in tension) can be made using rotating sensors as well [23]. However, most of these techniques are limited to a specific material and are inaccurate for large deflections [23]. Zhang et al. [24] first proposed a new method for extract the elastic modulus and residual stresses using nanoindentation. Specifically, SiN freestanding double clamped beams are deflected in the center by a nanoindenter. Then, FEM is then applied to extract the elastic modulus and residuals stresses, while accounting the support compliances and the bending moment. Zhou et al. [25, 26] extended the procedure for metallic $\mathrm{Ni}$ and $\mathrm{NiFe}$ double clamped beams, while Herbert et al. [27] improved the procedure to extract the elastic modulus and residual stress. Specifically, they developed an analytical solution that manages to accurately fit nanoindentation data under the assumption that the film deformation is dominated by stretching during indentation experiments [27]. Other assumptions consider the film flat, while the indent location is exactly in the center of the double clamped beam. The deflection is normal and elastic and the effect support posts are and the bending moments are ignored. In this model the residual stress are originated by the constrained beam geometry which prevent a full relaxation of residual stresses after releasing.

Bilayer double clamped beams have also been investigated due to the possibility to reduce the amount of residual stresses. Zhang et al. [28] and Nie et al. [29] used nanoindentation and pull-in methods respectively, to obtain the elastic moduli and residual stresses. However, a complex experimental set-up combined with FEM is required.

In this work, we use nanoindentation to study the mechanical properties of bilayer cantilevers and double clamped beams in order to extract elastic moduli and residual stress. Specifically, (i) we implemented the classic beam theory for a stress-free bilayer cantilever enabling the extraction of the elastic moduli of both layers using the stiffness measurement obtained by nanoindentation on different cantilever lengths. Then, (ii) we determine the residual stress by nanoindentation testing of double clamped beams, which are fabricated on the same $\mathrm{Si}$ wafer with the cantilevers, extending the 
analytical model of Herbert et al. [27] for a bilayer system and taking into account the indentation effect during loading.

We show that: (i) the obtained elastic moduli are in good agreement with TiW and Au films given by nanoindentation and with the average results given by Herbert's model [27]; (ii) the extracted residual stress are agreement with the values obtained from the double slot FIB and Digital Image Correlation (DIC) procedure, providing an alternative way to assess of residual stress estimates for composite double clamped beams.

\section{Experimental Details}

\subsection{Production and characterization of cantilever and double clamped beams}

Bilayer cantilevers and double clamped beams were produced on Si (100) substrate using standard microfabrication techniques involving substrate cleaning, lithography, sputter deposition and etching. Figure 1 is a Field Emission Gun Scanning Electron Microscope (FEG-SEM) image of the investigated cantilevers and double clamped beams. The shortest cantilever in Figure 1a is $20 \mu \mathrm{m}$-long, subsequent beams are incrementally $20 \mu \mathrm{m}$ longer up to a maximum of $260 \mu \mathrm{m}$ while their width was constant and equal to $10 \mu \mathrm{m}$. Three double clamped beams are present as well with a constant length and width equal to $280 \mu \mathrm{m}$ and $10 \mu \mathrm{m}$, respectively (right part of Figure 1a). The longest cantilevers are deflected toward the underneath substrate, while the shortest ones (from 20 up to $60 \mu \mathrm{m}$ ) and the double clamped beams remain perfectly straight (Figs. 1b and 1c). Figures 1c and 1d are SEM tilted views of the specimen showing the bilayer structure composed by a top Au layer (500 nm-thick) which uniformly covers the 200 nm-thick TiW layer.

Homogeneous $\mathrm{Au}$, TiW and Au/TiW films were produced on the same Si (100) substrate as well by using the same deposition parameters. 

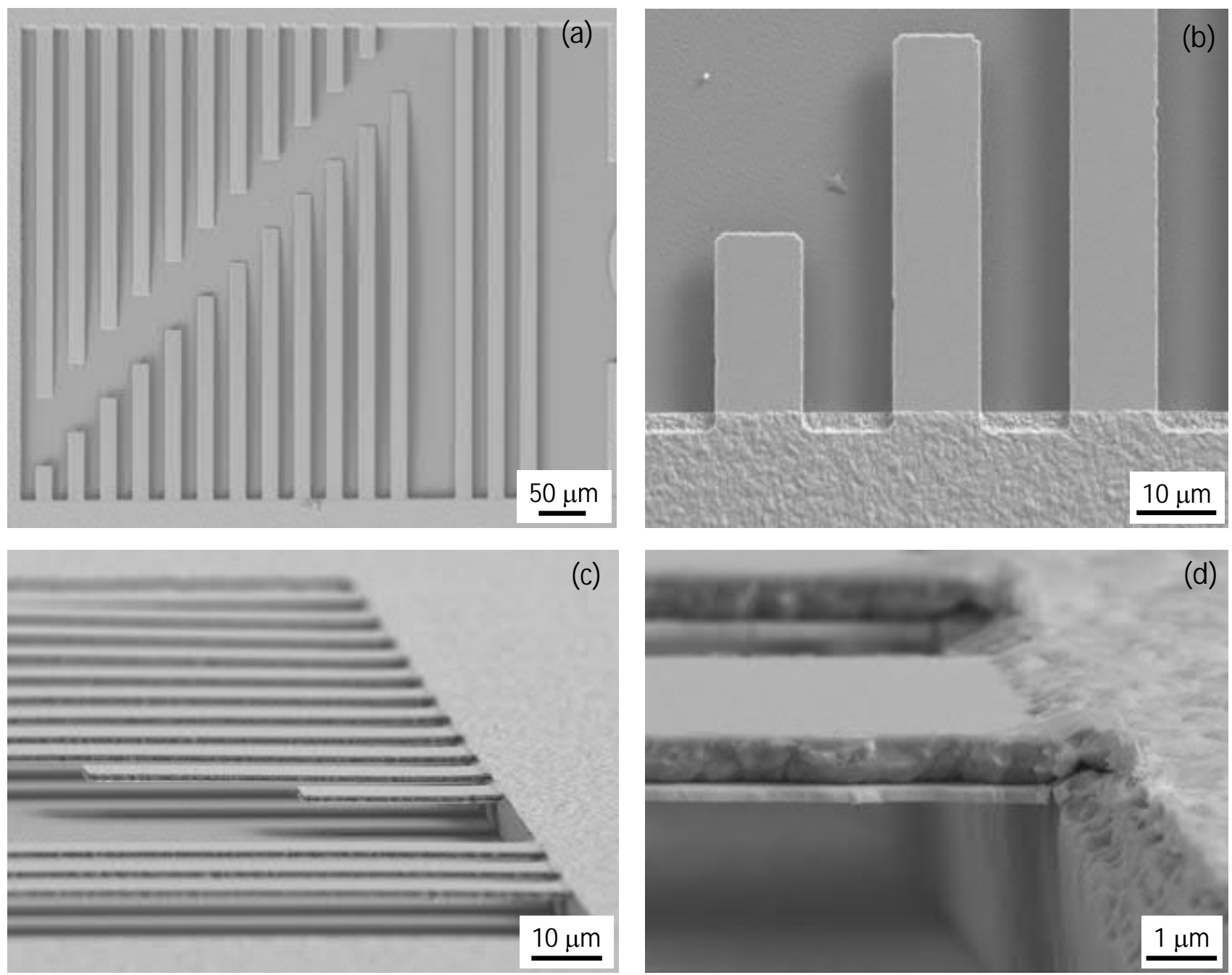

Fig. 1. SEM micrographs of cantilevers and double clamped beams. (a) The entire beam set showing deflection of the longest cantilevers. (b) A magnified image of the shortest cantilever showing no deflection. $(c, d)$ Tilted views highlighting the bilayer structure.

The 3D topography of the freestanding cantilevers and double clamped beams was obtained using a Leica microsystems DCM-3D non-contact optical profilometry in confocal mode. Figure 2a is 2D view acquired using optical profilometer highlighting the deflection of longer cantilevers. No curvature changes are observed for the shortest cantilever and double clamped beams, as also confirmed by the $3 \mathrm{D}$ view reported in Figure $2 \mathrm{~b}$. Figure $2 \mathrm{c}$ shows the extracted profile between the structures and the substrate underneath taken in the interval 1-2 in Figure 2a reporting a constant gap value equal to $3.8 \mu \mathrm{m}$. 

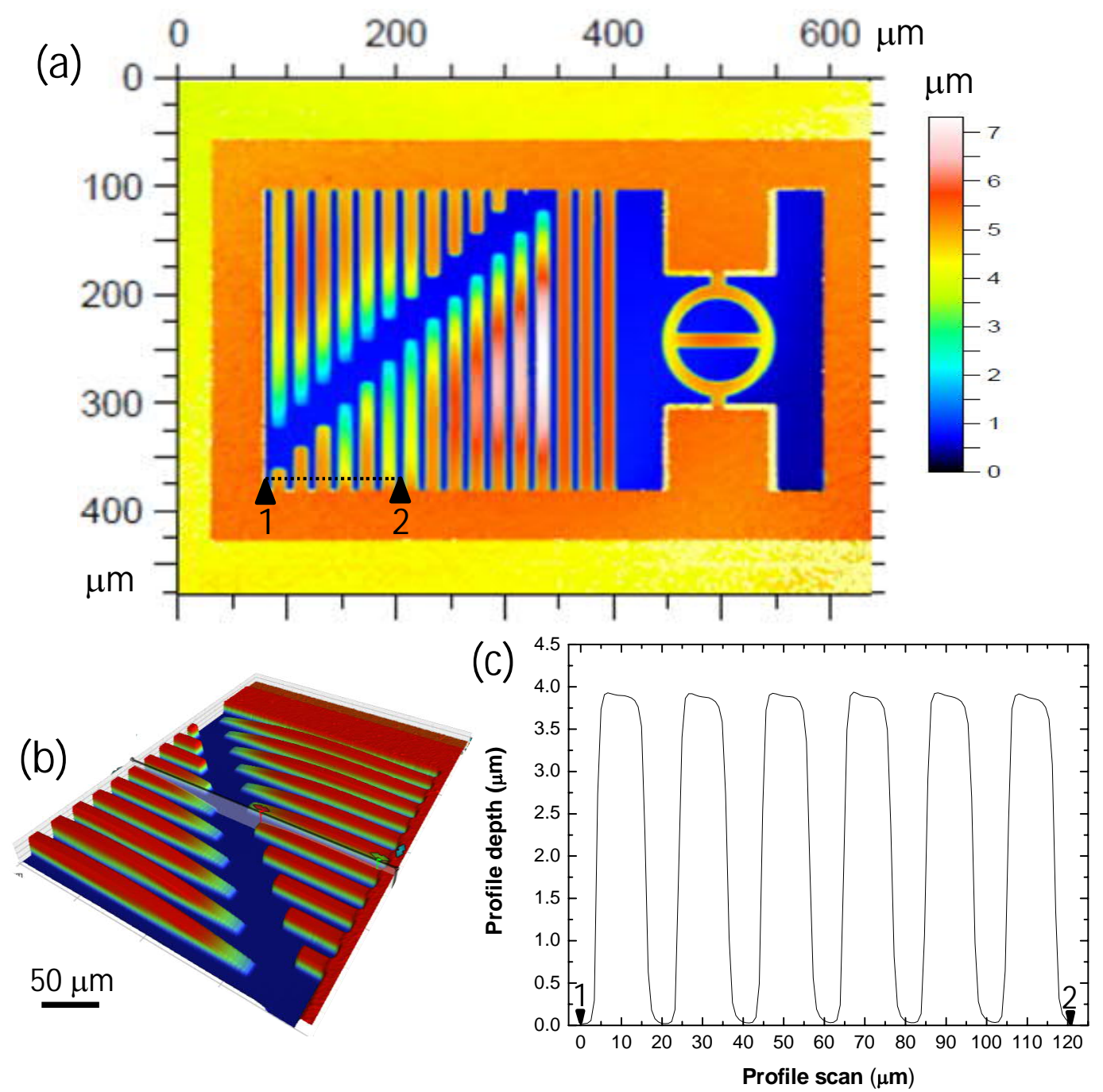

Fig. 2. Optical profilometer images of cantilevers and double clamped beam. (a) The entire beam set showing deflection of longest cantilevers. (b) 3D images of cantilevers. (c) Extracted thickness used to measure the gap with the substrate underneath $(\sim 3800 \mathrm{~nm})$.

FIB-SEM standard cross-sections were produced using a FEI Helios NanoLab 600 on the double clamped beams and had dimensions of $6 \mu \mathrm{m}$ in width and $15 \mu \mathrm{m}$ in length. Energy Dispersive X-ray Spectroscopy (EDS) was performed along the FIB-milled cross-section in order to distinguish different layers as well as to observe possible inter-diffusion phenomena (Figure 3a).The EDS mapping in Figure $3 b$ reveals sharp interfaces between each layer without evidences of interdiffusion effects. This confirms the nominal sequence and thicknesses of the layers (Au/TiW). The EDS spectra acquired in three different points within the cross-section is reported in Figure 3c. 

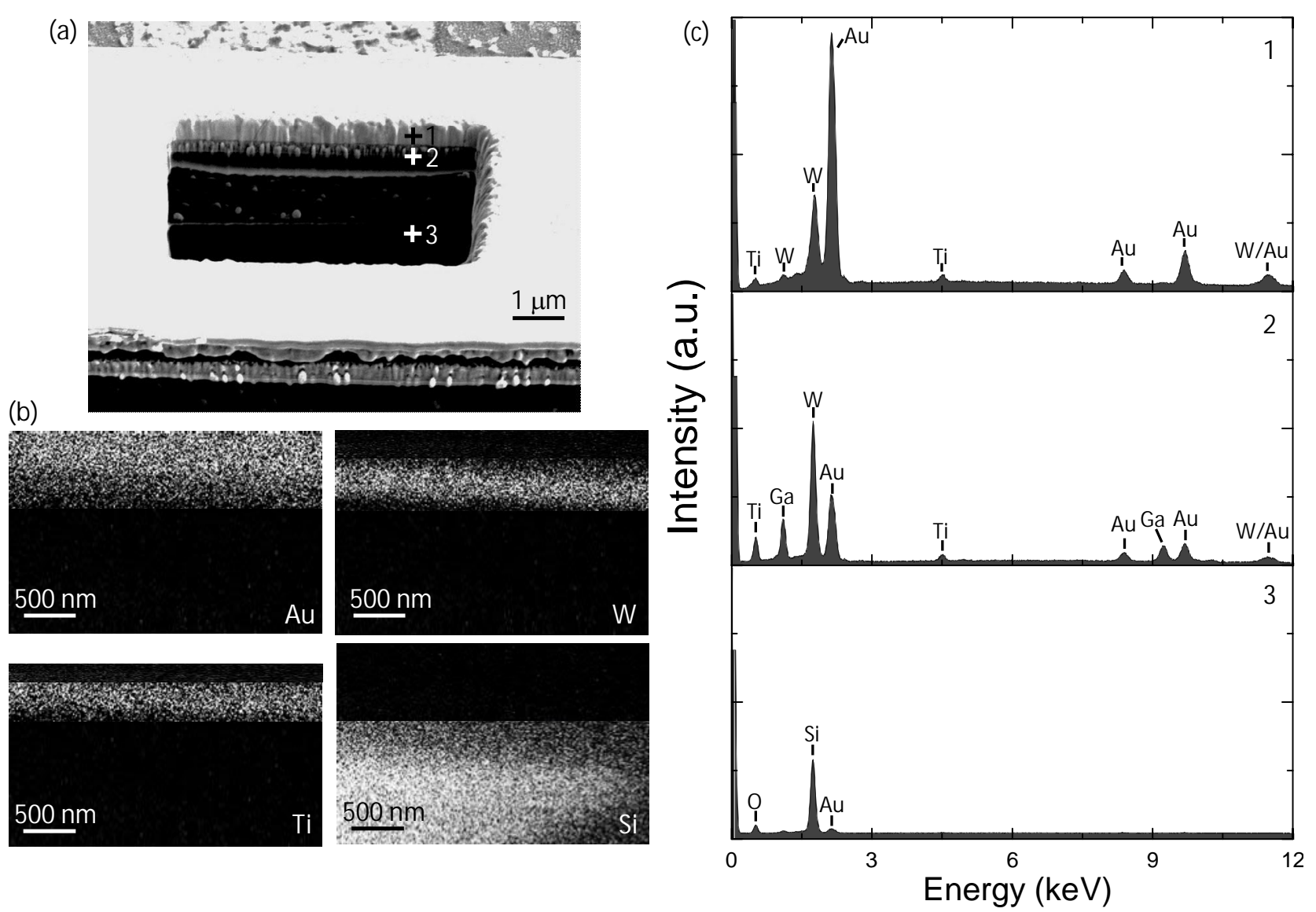

Fig. 3. EDS analyses of double clamped beams. (a) The FIB-milled cross-section. (b) The EDX mapping showing the Au-TiW bilayer structure. (c) The EDS spectra corresponding to layers 1, 2 and 3 in (a).

\subsection{Mechanical characterization of cantilever and double clamped beams}

a) Nanoindentation of uniform thin films on a substrate

Standard nanoindentation was used to extract the elastic modulus of the single homogeneous thin films on a Si (100) substrate. This technique also provided the load-depth curve for the $\mathrm{Au} / \mathrm{TiW}$ bilayer, which could be used to subtract the tip penetration effect occurring during deflection of double clamped beams.

Indentation tests were made with a diamond Berkovich tip mounted on an iNano nanoindenter (Nanomechanics Inc.). Prior to testing, the tip area function and frame stiffness were calibrated using a 
fused silica reference sample. The nanoindentation measurements were performed under load-control mode at room temperature using the Dynamic Stiffness Measurement (DSM) method [10]. The allowable thermal drift rate was limited to $0.05 \mathrm{~nm} \mathrm{~s}^{-1}$, while the imposed strain rate was set equal to $0.2 \mathrm{~s}^{-1}$. Sixteen indents were performed on each sample setting the maximum indentation depth equal to the film thickness. The elastic modulus of Au was extracted by fitting the raw indentation data using the King's model [30] in order to avoid substrate effect.

\section{b) Nanoindentation of freestanding cantilevers and double clamped beams}

Cantilevers and double clamed beams were tested by nanoindentation in DS mode as well using a diamond Berkovich tip mounted on an iNano nanoindenter (Nanomechancs Inc.). The maximum load was equal to $1 \mathrm{mN}$ with a linear loading rate applied. The surface approach distance - defined as the starting distance between the tip and the substrate- was fixed equal to $6000 \mathrm{~nm}$, being the structures suspended on underneath substrate of about $4000 \mathrm{~nm}$ (Section 2.1). Hence, prior to indentation the indent was located $2000 \mathrm{~nm}$ above the surface in order to avoid lateral contact between the tip and the testing structure. A microscope to indenter calibration was performed before each test in order to avoid a non-uniform bending with possible torsional effects. All cantilevers were indented at $5 \mu \mathrm{m}$ from the free edge, while $20 \mu \mathrm{m}$-spaced indents were made on double clamped beams along the entire length. The indenter position was additionally verified a posteriori by observing the indent location at the optical profilometer and by evaluating the quality of the test itself. The output provided, apart from the load-displacement curve, the stiffness of the beam and the gap to the substrate. The DS is continuously recorded as function of the beam deflection. The gap, namely the distance which separate the beams with the underneath substrate, was provided by detecting an abrupt change of stiffness at a certain depth indicating the substrate detection. The effects of supporting spring stiffness and indenter 
penetration into the double clamped beams were properly taken into account and corrected according to previous literature works $[12,27]$.

\section{c) Independent residual stress estimates by FIB-DIC double slot milling}

In order to have comparative estimates of residual stress profiles in the bilayer systems, residual stresses were measured by using FIB double slot milling method, coupled with DIC for relaxation strain mapping. As reported in a recent paper [31], two parallel slots were FIB milled in a stepwise fashion, along the perpendicular direction with respect to beam axis, so to induce incremental relaxation of the residual stress. The resultant relaxation strain profiles were measured by DIC in the central area, after acquiring a series of high-resolution SEM images, before and after each milling step.

A low current $(9 \mathrm{pA})$ were used during FIB milling to acquire an accurate longitudinal strain profile as a function of the milling depth in both layers, and to minimize the ion-induced damage.

The distance between two contiguous experiments was set to $10 \mu \mathrm{m}$, being the internal distance between the slots equal to $3 \mu \mathrm{m}$.

As demonstrated in Ref. [31], double slot milling geometry is characterized by an almost uniaxial relaxation strain, that is representative of the average stress along the beam principal direction in this specific case. The residual stress $\left(\sigma_{r}\right)$ in the films were finally calculated from measured relaxation strains by using FEM, as reported in previous papers [31, 32]. Specifically,

$$
\sigma_{r}=-\frac{E \cdot \varepsilon_{r}}{k}
$$

where $E$ is the elastic modulus of the film, $\varepsilon_{r}$ is the measured relaxation strain extracted at a depth of 500 and $700 \mathrm{~nm}$ for $\mathrm{Au}$ and TiW layer, respectively and $k$ is a FEM calculated constant equal to 1.47 
for the geometry used in this work, where the length of the slots is equal to the distance between them $[31,32]$.

\section{Procedure to extract the elastic moduli and residual stresses for bilayer structures}

According to the beam theory [33], the measurement of stiffness in a freestanding cantilever can be used to calculate its elastic modulus. Specifically, for rectangular cross-sections, the load at the anchoring $(P)$ is defined as

$$
P=\frac{3 I E}{l^{3}} h=\frac{w t^{3} E}{4 l^{3}} h,
$$

where $I$ is the moment of inertia, $E$ is the elastic modulus, $h$ the deflection, $l$ is the length corresponding to the position in which the deflection is performed, while $w$ and $t$ are the width and the thickness, respectively. The stiffness $(S)$ will be

$$
S=\frac{d P}{d h}=\frac{3 I E}{l^{3}}=\frac{w t^{3} E}{4 l^{3}} .
$$

Note that Equations (2) and (3) do not consider the bilayer structure of the beam, yet. The elastic modulus of each layer was determined by implementing the classic beam theory for a bilayer microcantilevers using the transformed section method $[17,33]$. The procedure is to transform the crosssection, consisting of a more than one material, into an equivalent cross-section composed of only one material. This leads to the definition of a new cross section composed of only one material, as show in Figure 4. Then, the beam with transformed section was analyzed using the standard beam theory [33]. Figure 4 shows that the second layer has been normalized respect to the elastic modulus of the top layer, so that the width of the second layer $\left(w_{2}\right)$ is

$$
w_{2}=\frac{E_{2}}{E_{1}} w=n \cdot w,
$$


where $n$ is the so-called modular ratio [33]. The new transformed beam is equivalent to the original beam with its neutral axis in the same position (dashed lines in Figure 4). However, the overall bending moment $\left(E I_{t}\right)$ will be

$$
E I_{t}=E_{1} I_{1}+E_{2} I_{2}
$$

where $I_{1}$ and $I_{2}$ are the moment of inertia of the first and second layer, respectively.

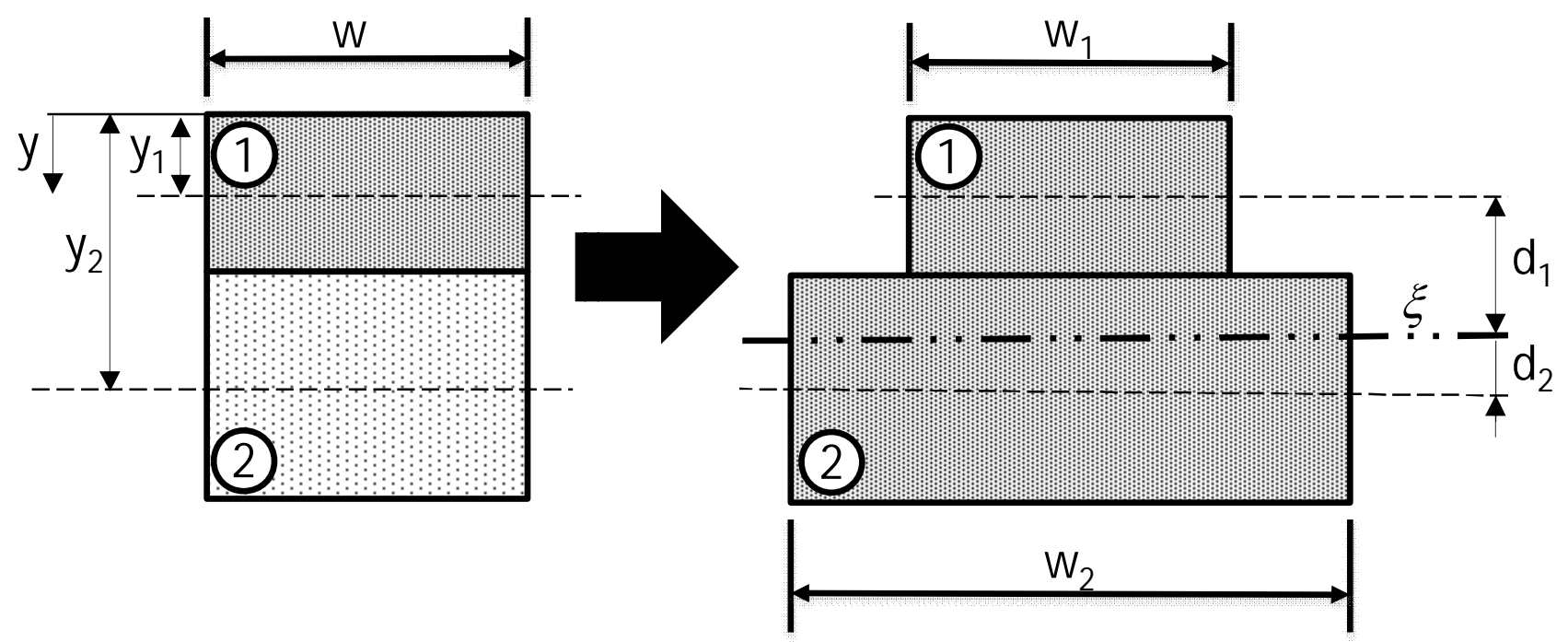

Fig. 4. The transformed section method. Beams of two different materials are transformed into a single material section with the same elastic modulus.

In the case of rectangular cross-section $I_{1}$ and $I_{2}$ can be written as

$$
I_{1}=\frac{w t_{1}^{3}}{12}+A_{1} d_{1}^{2} \text { and } I_{2}=\frac{w t_{2}^{3}}{12}+A_{2} d_{2}^{2} \text {, }
$$

where $A$ is the cross sectional area and $d$ the distance between the centroid axis $(\xi)$ of the composite beam and the neutral axis of each individual layer $(d=\xi-y)$, Figure 4 . The location of $\xi$ within the transformed section is given by

$$
\xi=\frac{\left(y_{1} A_{1}+y_{2} A_{2}\right)}{\left(A_{1}+A_{2}\right)}
$$


in which $y$ is the position of neutral axis of each layer with respect to top the surface (Figure 4). By inserting Equation (7) in Equations (6) and expanding all the terms, $I_{1}$ and $I_{2}$ are

$$
\begin{aligned}
& I_{1}=\frac{w t_{1}^{3}}{12}+w \cdot t_{1}\left[\frac{\left(y_{1} \cdot w \cdot t_{1} \cdot+y_{2} \cdot \frac{E_{2}}{E_{1}} \cdot w \cdot t_{2}\right)}{\left(w \cdot t_{1}+\frac{E_{2}}{E_{1}} \cdot w \cdot t_{2}\right)}-y_{1}\right]^{2} \\
& I_{2}=\frac{w t_{2}^{3}}{12}+\left(\frac{E_{2}}{E_{1}} \cdot w \cdot t_{2}\right)\left[\frac{\left(y_{1} \cdot w \cdot t_{1}+y_{2} \cdot \frac{E_{2}}{E_{1}} \cdot w \cdot t_{2}\right)}{\left(w \cdot t_{1}+\frac{E_{2}}{E_{1}} \cdot w \cdot t_{2}\right)}-y_{2}\right]^{2},
\end{aligned}
$$

in which the dependency of the moment of inertia with the elastic moduli of each layer is highlighted. In order to limit the inaccuracy of indent positioning, Equation (2) was set equal to 0 for a set of different cantilever lengths. This is equivalent to solve a system of Equations in which $E_{1}$ and $E_{2}$ are unknown

$$
\left\{\begin{array}{l}
E_{1} \cdot I_{1}\left(E_{1}, E_{2}\right)+E_{2} \cdot I_{2}\left(E_{1}, E_{2}\right)-\frac{S_{A} x_{A}^{3}}{3}=0 \\
E_{1} \cdot I_{1}\left(E_{1}, E_{2}\right)+E_{2} \cdot I_{2}\left(E_{1}, E_{2}\right)-\frac{S_{B} x_{B}^{3}}{3}=0 \\
E_{1} \cdot I_{1}\left(E_{1}, E_{2}\right)+E_{2} \cdot I_{2}\left(E_{1}, E_{2}\right)-\frac{S_{C} x_{C}^{3}}{3}=0 \\
\ldots
\end{array}\right.
$$

where the dependency on the momenta of inertia on the elastic moduli is highlighted. The set of Equations (11) was iterated up to convergence, leading to the extraction of $E_{1}$ and $E_{2}$. Specifically, the stiffness were provided by three different cantilever lengths $(20,40$ and $60 \mu \mathrm{m})$, and each measurement was repeated in order to provide improved statistical estimates.

The extraction of mechanical properties for freestanding double clamped beams was performed using the model of Herbert et al. [27]. This model relates the load $(P)$ with the penetration depth $(h)$ as 


$$
P=\frac{8 w t E h^{3}}{l^{3}}-\frac{8 w t \sigma_{r} h^{3}}{l^{3}}+\frac{4 w t \sigma_{r} h}{l},
$$

where $l$ is the length of the double clamped beams, while $E$ and $\sigma_{r}$ represent, respectively, the elastic modulus and the residual stresses generated by the constrained geometry. The stiffness is obtained as

$$
S=\frac{d P}{d h}=\frac{24 w t E h^{2}}{l^{3}}-\frac{24 w t \sigma_{r} h^{2}}{l^{3}}+\frac{4 w t \sigma_{r}}{l} .
$$

Hence, by fitting the trend of the stiffness as a function of the depth it is possible to extract the elastic modulus and the residual stresses for a monolayer. Contrary to cantilevers which have a free edge, the constrained geometry of double clamped beams enables the generation of residual stresses as also reported in Refs. [21, 22] using similar testing structures.

In order to extract the residual stresses of each layer using Herbert's model, the elastic moduli obtained from the cantilever bending were used. The main hypothesis is that both layers are rigidly attached (iso-deformation condition). Hence, the effective elastic modulus $\left(E_{e f f}\right)$ and the residual stresses $\left(\sigma_{r}^{e f f}\right)$ are

$$
E_{\text {eff }}=\frac{A_{1} E_{1}+A_{2} E_{2}}{A}
$$

and

$$
\sigma_{r}^{e f f}=\frac{A_{1} \sigma_{r 1}+A_{2} \sigma_{r 2}}{A}
$$

By inserting the Equations (13) and (14) into Equations (11) and (12), the load and the stiffness become

$$
\left\{\begin{array}{l}
\frac{8\left(A_{1} E_{1}+A_{2} E_{2}\right) h^{3}}{l^{3}}-\frac{8\left(A_{1} \sigma_{r 1}+A_{2} \sigma_{r 2}\right) h^{3}}{l^{3}}+\frac{4\left(A_{1} \sigma_{r 1}+A_{2} \sigma_{r 2}\right) h}{l}-P=0 \\
\frac{24\left(A_{1} E_{1}+A_{2} E_{2}\right) h^{2}}{l^{3}}-\frac{24\left(A_{1} \sigma_{r 1}+A_{2} \sigma_{r 2}\right) h^{2}}{l^{3}}+\frac{4\left(A_{1} \sigma_{r 1}+A_{2} \sigma_{r 2}\right)}{l}-S=0
\end{array}\right.
$$


The convergence of Equation (15) was computed for $\sigma_{r 1}$ and $\sigma_{r 2}$ by using an iterative routine which exploits the entire set of data points acquired during the deflection (true contact range) in order to provide highly accurate results. The indent penetration during deflection was also subtracted.

\section{Results and discussion}

\subsection{Extraction of elastic moduli by cantilever testing}

Figure 5 reports the mechanical characterization of shortest three cantilevers, namely 20,40 and $60 \mu \mathrm{m}$ which do not show any deflection (Section 2.1). All bending tests have been made at $5 \mu \mathrm{m}$ from the free edge. In Figures 5a-c the stiffness is plotted as a function of the indentation depth. Once the indent touches the cantilever, the stiffness is immediately detected. The extracted value decreases from $31 \mathrm{~N} / \mathrm{m}$ for down to $1.7 \mathrm{~N} / \mathrm{m}$, respectively for 20 and $60 \mu \mathrm{m}$-long cantilevers. The extraction of the stiffness for cantilevers longer than $60 \mu \mathrm{m}$ was not possible, because the acquired value is below the noise associated to measurement $(\sim 1 \mathrm{~N} / \mathrm{m})$. In order to acquire more statistics, measurements have been repeated for other cantilever sets locating the indent at the same distance from the anchoring. Average stiffness results for 20, 40 and $60 \mu \mathrm{m}$-long cantilevers obtained on two independent set of measurements are reported in Figure 5d indicating good reproducibility of the data. After the detection of the stiffness, the cantilevers are bent towards the substrate, whose contact is indicated by the abrupt stiffness increase. It is worth noting that the stiffness is not affected by the penetration of the indenter in the cantilever since it is extracted at almost zero depth, namely when the indenter detects the cantilever surface. Moreover, the measurement of the gap between the cantilevers and the substrate underneath is not very accurate for short cantilevers, because of the indent gliding effects. Figure $5 \mathrm{~d}$ reports the average stiffness as a function of the indentation $x$-coordinate, namely the position on which the bending is performed. The data points have been overlapped using the stiffness data calculated with 
Equation (3) with an effective elastic modulus equal to $108 \mathrm{GPa}$, a value extracted using the Herbert model [27], while assuming the double clamped beams as a single layer. Figure 5d shows that the stiffness increases when indenting close to the cantilever anchoring as expected using the standard beam theory [33], Equation (3). The accurate overlapping between the curve and the data points in Figure $5 \mathrm{~d}$ is a further prove of (i) the precise indent positioning and of (ii) the average elastic modulus values obtained using two different techniques and analytical models.
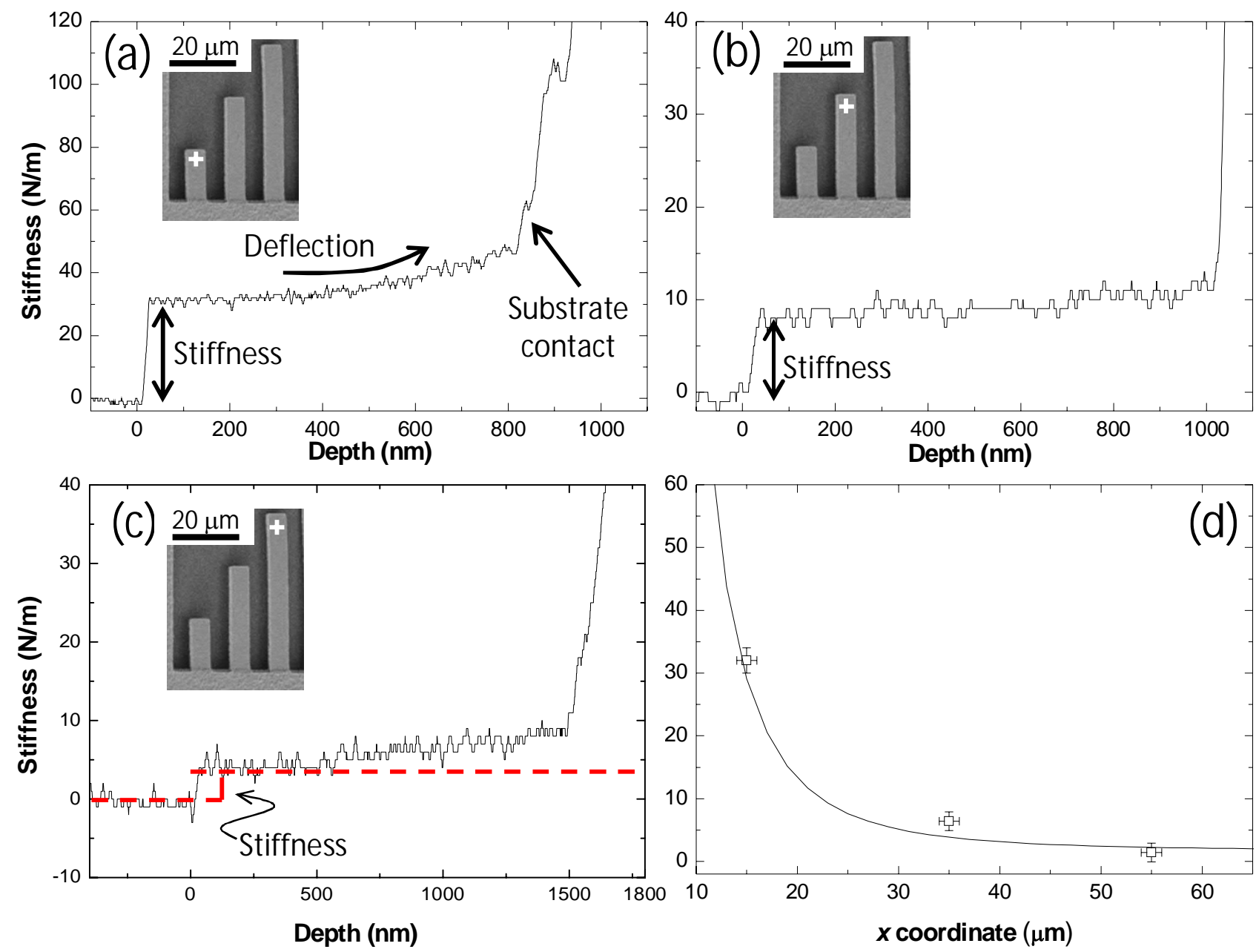

Fig. 5. Stiffness analyses for cantilevers with different lengths. (a)-(c) Stiffness as a function of the indentation depth for 20, 40 and $60 \mu$ m-long cantilevers, respectively. (d) Stiffness as a function of the indent position on the cantilever. Data are overlapped with the results of Equation (3) using an elastic modulus equal to $108 \mathrm{GPa}$ (continuous line). 
By taking the stiffness values in Figure 5d for two different set of cantilevers with a length equal to 20, 40 and $60 \mu \mathrm{m}$, and applying the transformed section method (Section 3), we found that Equation (10) convergences toward $E_{1}$ and $E_{2}$ values respectively equal to $74 \pm 8$ and $232 \pm 8 \mathrm{GPa}$. Note that this procedure reduces the experimental error associated to the calculation, because the solution is obtained from 6 different cantilevers with length varying from 20 up to $60 \mu \mathrm{m}$. The obtained results are in agreement with the literature values for $\mathrm{Au}[34,35]$ and TiW [36] films (Table 1). Moreover, these values are consistent with the average ones obtained using Equation (12) for double clamped beams providing an average elastic modulus equal to $108.5 \mathrm{GPa}$ (Section 4.2).

Table 1. Summary of the obtained elastic moduli for $A u$ and TiW.

\begin{tabular}{lcc}
\hline & $E_{A u}(\mathrm{GPa})$ & $E_{T i W}(\mathrm{GPa})$ \\
\hline Literature & $79[34]$ & $260[36]$ \\
Cantilever bending & $74,110[35]$ & $232 \pm 8$ \\
Nanoindentation & $80 \pm 5$ & $219 \pm 20$ \\
\hline
\end{tabular}

Figure 6a shows the elastic moduli as a function of the normalized indentation depth for Au and TiW films on $\mathrm{Si}(100)$ substrate. The elastic modulus of $\mathrm{Au}$ increases from $83 \pm 20 \mathrm{GPa}$ for small indentations up to $180 \pm 3 \mathrm{GPa}$ converging toward the Si (100) values (183 GPa). The Au elastic modulus has been extracted by fitting the indentation depth data using the King's model (Figure 6b) founding a value equal to $80 \mathrm{GPa}$ in agreement with literature values $[34,35]$ and the results obtained using the transformed section method applied to the bilayer cantilever deflection (Table 1). The TiW elastic modulus calculated between 20 and $50 \mathrm{~nm}$-depth is equal to $219 \pm 20 \mathrm{GPa}$, in agreement with both literature [36] and bilayer cantilever bending results (Table 1). As expected, results show the 
absence of mechanical size effects on the elastic moduli, as also demonstrated in other literature studies on metallic glasses [4, 37] and Au micro-pillars [34].
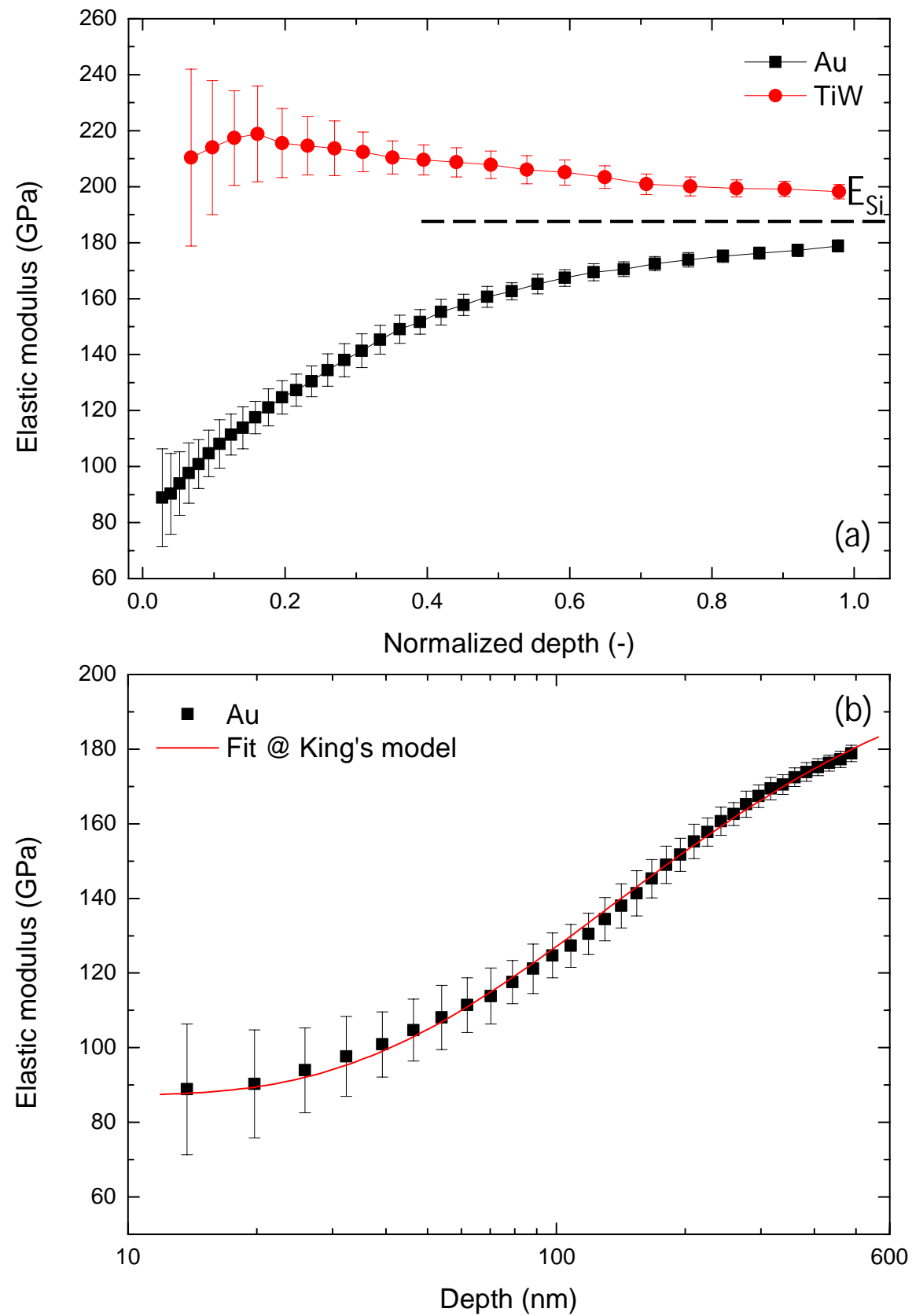

Fig. 6. (a) Elastic modulus as a function of the normalized indentation depth for TiW and Au thin films on a Si (100) substrate. (b) Elastic modulus of Au as a function of the indentation depth. The data was fitted using the King's model [30] to extract the elastic modulus of the film (continuous red line). 


\subsection{Extraction of residual stress by double clamped beam deflection}

The extraction of residual stresses has been made by deflection of double clamped beams. As a matter of fact, cantilevers have one free edge thus resulting in no residual stresses. Figure 7a show the stiffness profile as a function of the $x$-coordinate within the double clamped beam. Ten indentations, separated by $20 \mu \mathrm{m}$, have been made along the double clamped beam. As expected, the stiffness shows a symmetric parabolic profile with the maximum values $(6.2 \pm 0.3 \mathrm{~N} / \mathrm{m})$ close to the anchoring points, while the minimum values $(2.3 \pm 0.2 \mathrm{~N} / \mathrm{m})$ is recorded when indentation is performed in the middle of the beam. Figure $7 \mathrm{~b}$ reports the trend of the stiffness as a function of the indentation depth when performing a deflection test in the middle of the beam (inset of Figure 7b). After the initial contact, the deflection starts following a parabolic profile, as observed in previous papers [27], equation (12). The substrate is detected when an abrupt increase of stiffness is observed. The gap between the beam and the substrate underneath has been found equal to $3850 \mathrm{~nm}$ in agreement with optical profilometer results (Figure 3c). The same gap value is found on the load versus depth curve as well, Figure 7c. Note that at larger depths the effect of the indent penetration in the beam further enhance measured stiffness. The elastic modulus and residual stress have been extracted using the model of Herbert et al. [27]. The double clamped beam has been considered as constituted by a single layer, then the stiffness data in the contact range have been fitted using Equation (12), red dashed line. The average elastic modulus is equal to $108 \mathrm{GPa}$ and the average residual stress are equal to $+31 \mathrm{MPa}$ in tension. The residual stress for each layer is extracted using Equations (15). Specifically, we used the elastic moduli equal to 74 and $232 \mathrm{GPa}$ as extracted from cantilever deflection and we iterate Equation (15) over the entire contact range (identified by the vertical dashed lines on Figures $7 \mathrm{~b}$ and $7 \mathrm{c}$ ). We found that residual stresses are equal to $+6.9 \pm 3$ and $+56.6 \pm 5 \mathrm{MPa}$, respectively for the $\mathrm{Au}$ and TiW layers (Table 2). In order to acquire very accurate values of residual stresses, the beam deflection has been corrected by the indent penetration. 

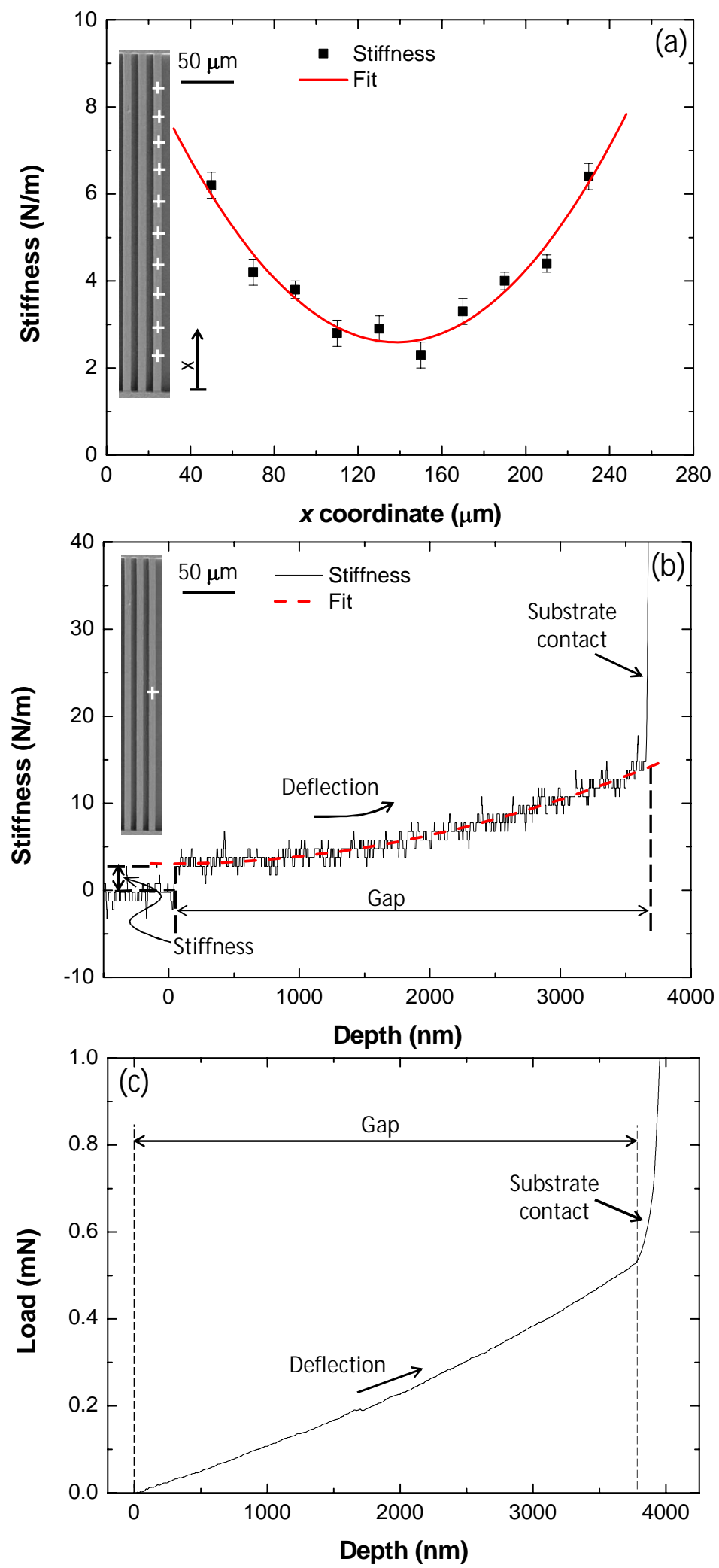

Fig. 7. Stiffness analyses for double clamped beams. (a) The stiffness as a function of the x-coordinate along the beam, highlighting a parabolic trend. (b) The stiffness versus depth for a double clamped beam deflected in the center. A parabolic fit with Equation (11) has been performed, red dashed line. (c) The load as a function of depth for a double clamped beam deflected in the center. 
Specifically, Figure 8 reports the load-depth curve obtained when indenting the Au/TiW layer on Si (100) substrate, which are used to correct for indenter penetration during tests on suspended double clamped beams. It is worth to note that at $1 \mathrm{mN}$ (i.e. the maximum applied deflection load) the penetration of the indenter in the double clamped beams is around $110 \mathrm{~nm}$ (Figure 8).

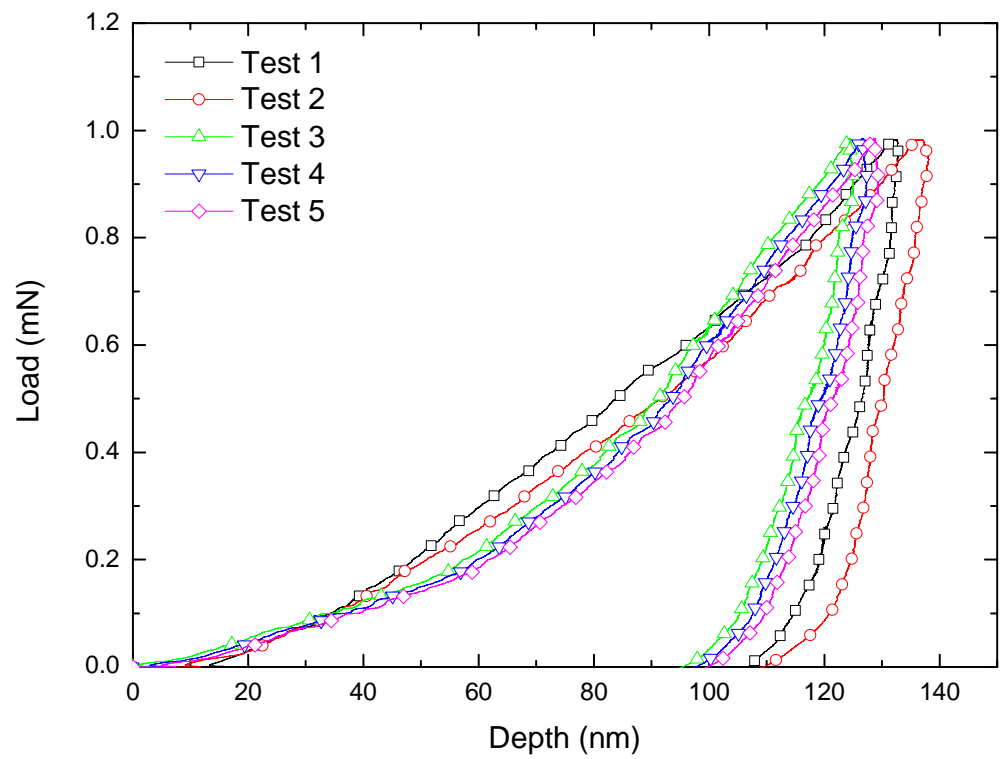

Fig. 8. The load-depth curves for Au-TiW layers on a Si (100) substrate which were used to subtract the indentation effect during double clamped beam deflection.

In the case of homogeneous $\mathrm{Au}$ uniform thin films, the residual stress estimates range from the compressive to the tensile region, depending on the substrate nature and the process parameters [3739]. Differences with respect to uniform thin film are due to the freestanding geometry of double clamped beams in which most of the residual stresses are released after substrate removal. In the case of Au micro-beams a tensile residual stress has been reported in between +14 and $+33 \mathrm{MPa}$, Ref. [38, 39] which is closer to our results (Table 2). A comparison of residual stress for TiW is more difficult because the lake literature works on this material. However, high tensile residual stresses are expected because the ceramic-like nature of intermetallic TiW alloys [40]. It is worth to note that the average residual stress values of both layers using Equation (14) gives an overall value of equal to $+32 \mathrm{MPa}$, in agreement with the $+31 \mathrm{MPa}$ provided by the Herbert et al. model [27] applied for monolayer, 
Equation (12). Equations (15) have also be solved using four independent variables, namely the residual stresses and the elastic moduli corresponding to each layer. This provides an elastic modulus equal to 82 and $222 \mathrm{GPa}$ and residual stresses equal to +10 and $+50 \mathrm{MPa}$, respectively for Au and TiW. Although this procedure is less accurate because four independent variables are iterated, the results are very close to the ones obtained using the elastic moduli obtained from bending test of cantilevers, confirming the quality of the adopted methodology.

\subsection{Validation by FIB-DIC residual stress measurements}

FIB-DIC double slot experiments have been carried out in order to confirm the value of residual stresses determined using nanoindentation. Figure 9a and the zoom in Figure 9b show the two slots which are milled along the perpendicular direction with respect to beam axis. The trend of the relaxation strain, extracted using the DIC, is reported as a function of the milling depth in Figure 9c. Specifically, the relaxation strain oscillates around zero for the Au layer, while decreasing down to -

$6.5 \cdot 10^{-4} \pm 10^{-4}$ for the TiW layer. The residual stress in the Au layer, calculated at a milling depth equal to $500 \mathrm{~nm}$ using Equation (1), is equal to $-1.6 \pm 5 \mathrm{MPa}$ indicating the residual stress in the Au are almost negligible. However, the measured value is close to the resolution limit of the technique used to measure the relaxation strain [31]. On the other hand, TiW exhibits a clear tensile residual stress which is equal $+102 \pm 16 \mathrm{MPa}$. Both results are in a very good agreement with the extracted residual stress obtained by bending test of double clamped beams (Table 2). However, it is worth noting that the assumptions made when extracting the residual stress using nanoindentation of bilayer double clamped beams do not allow compressive residual stresses, the interfaces between the layers are assumed perfect and the surface roughness is not taken into account. This possibly explains the differences between the two methodologies and will be the subject of future investigations. 

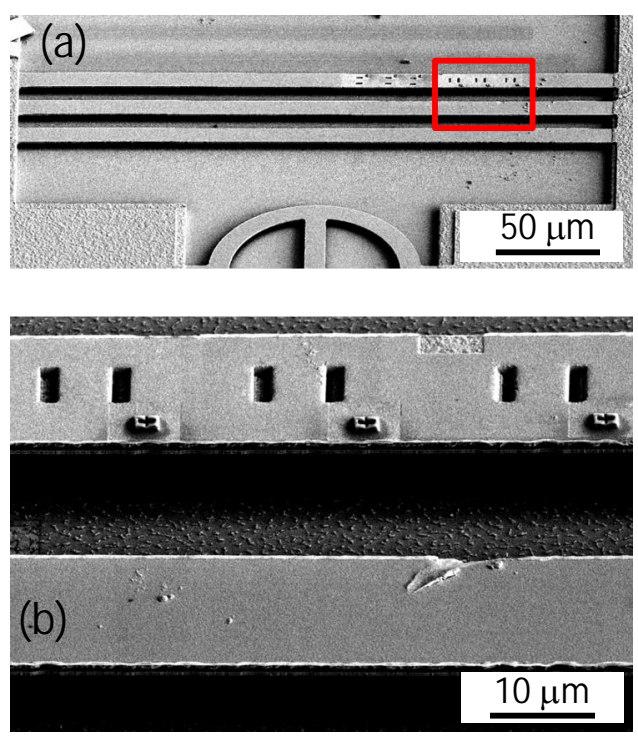

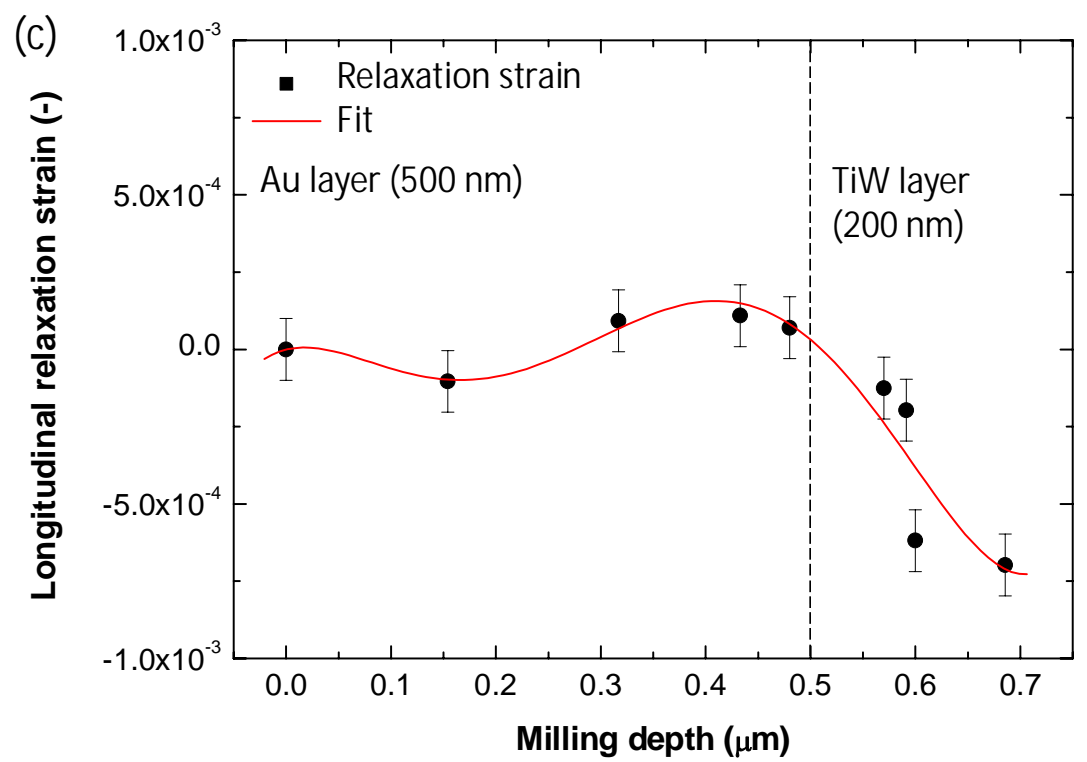

Fig. 9. Extraction of residual strains using FIB-DIC. (a) The two slots milled perpendicular to the double clamped beam direction. (b) A magnified image showing the double-slot FIB milling. (c) The relaxation strains as a function of the milling depth showing negligible residual strain for Au layer and a more pronounced tensile strain for TiW layer. Data were fitted with a fifth degree polynomial function (red lines) and then used for calculating the average stresses in the two layers.

Table 2 summarizes the obtained results for residual stresses for Au and TiW. Specifically, it can be noted that the results obtained with our methodology - involving bending test on freestanding cantilevers and double clamped beams - enable to accurately extract residual stresses for a bilayer system, providing an easy procedure which can be adopted to determine a key mechanical property of MEMS.

Table 2. Summary of the residual stress estimates for Au and TiW.

\begin{tabular}{lcc}
\hline & $\sigma_{r, A u}(\mathrm{MPa})$ & $\sigma_{r, T i W}(\mathrm{MPa})$ \\
\hline Literature & $+14.1,+33.3[38]$ & - \\
$\begin{array}{l}\text { Double clamped } \\
\text { beams bending }\end{array}$ & $+6.9 \pm 3$ & $+56.6 \pm 5$ \\
$\begin{array}{l}\text { Double slot FIB- } \\
\text { DIC }\end{array}$ & $-1.6 \pm 7$ & $+102 \pm 16$ \\
\hline
\end{tabular}




\section{Conclusions}

In this work, we have presented an innovative experimental protocol for the measurement of elastic moduli and residual stresses in by-layer suspended micro-beams.

By combining nanoindentation deflection experiments on both cantilevers and double-clamped beams of an Au/TiW system, we develop a fully analytical model for the simultaneous extraction of the elastic moduli and residual stresses in the two layers.

The measurements on bilayer double clamped beams and cantilevers have been validated by comparing the results with (i) elastic moduli obtained by nanoindentation experiments on analogous homogeneous films on Si substrate and (ii) with the residual stresses obtained by FIB-DIC double slot measurements on the same double clamped beams.

Further experiments will be required to extend the method to systems where more than two layers are present and to those cases in which a compressive stress is present in one of the layers.

The developed procedures are directly scalable to the MEMS industry quality control level and can be extremely important for supporting the design and optimization of novel micro-devices with improved performance not only RF-switches, but also accelerometers, pressure sensors and other kind of MEMS.

\section{Acknowledgements}

Authors thank Ms. Fabrizia Vallerani for technical assistance during FIB experiments at the interdepartmental laboratory of electron microscopy (LIME) of "Roma TRE" University in Rome, Italy. The financial support for this work was provided through the European FP7 Project, iSTRESS (Grant agreement \# 604646,www.istress.eu). 


\section{References}

[1] Franssila S, Introduction to Microfabrication. 2010: John Wiley \& Sons Ltd.

[2] Greer JR and JTM De Hosson. Plasticity in small-sized metallic systems: Intrinsic versus extrinsic size effect. Prog. Mater. Sci. 2011;56:654-724.

[3] Artz E. Size effects in materials due to microstructural and dimensional constraints: a comparative review. Acta Metall. 1998;46:5611-5626.

[4] Ghidelli M, S. Gravier, J.-J. Blandin, P. Djemia, F. Mompiou, G. Abadias, J.-P. Raskin, and T Pardoen. Extrinsic mechanical size effects in thin $\mathrm{ZrNi}$ metallic glass films. Acta Mater. 2015;90:232-241.

[5] Espinosa HD, B.C.Prorok, and M.Fischer. A methodology for determining mechanical properties of freestanding thin (lms and MEMS materials. J. Mech. Phys. Solids. 2003;51:4767.

[6] Zeng $\mathrm{H}$ and W Sharpe Jr. A system for measuring biaxial creep strains over short gage lengths. Exp. Mech. 1996;36:84-91.

[7] Vlassak J and W Nix. A new bulge test technique for the determination of Young's modulus and Poisson's ratio of thin films. J. Mater. Res. 1992;7:3242-3249.

[8] Boé A, A Safi, M Coulombier, T Pardoen, and JP Raskin. Internal stress relaxation based method for elastic stiffness characterization of very thin films. Thin Solid Films. 2009;518:260264.

[9] Nix WD. Mechanical Properties of Thin Films. Metall. Trans. A. 1989;20A:2217-2245. 
[10] Oliver WC and GM Pharr. Measurement of hardness and elastic modulus by instrumented indentation: Advances in understanding and refinements to methodology. J. Mater. Res. 2003;19:3-20.

[11] Weihs TP, S Hong, JC Bravman, and WD Nix. Mechanical deflection of cantilever microbeams: A new technique for testing the mechanical properties of thin films. J. Mater. Res. 1988;3:931-942.

[12] Tsou C. Measuring thin film elastic modulus using a micromachined cantilever bending test by nanoindenter. Journal of Micro/Nanolithography, MEMS, and MOEMS. 2007;6:033011.

[13] Gong $\mathbf{J}$ and $\mathrm{A}$ Wilkinson. Investigation of elastic properties of single-crystal $\alpha$-Ti using microcantilever beams. Philod. Mag. Lett. 2010;90:503-512.

[14] Guo Q, L Zhang, AS Zeiger, Y Li, KJ Van Vliet, and CV Thompson. Compositional dependence of Young's moduli for amorphous $\mathrm{Cu}-\mathrm{Zr}$ films measured using combinatorial deposition on microscale cantilever arrays. Scr. Mater. 2011;64:41-44.

[15] Liu Y, S Hata, K Wada, and A Shimokohbe. Thermal, mechanical and electrical properties of Pd-based thin-film metallic glass. Jpn. J. Appl. Phys. 2001;40:5382-5388.

[16] Florando JN and WD Nix. A microbeam nending method for studying stress-strain relations for metal thin films on silicon substrates. J. Mech. Phys. Solids. 2005;53:619-638.

[17] Boyd E, V Nock, D Weiland, X Li, and D Uttamchandani. Direct comparison of stylus and resonant methods for determining Young's modulus of single and multilayer MEMS cantilevers. Sensors Actuat. A. 2011;172:440-446.

[18] Fang W. Determination of the elastic modulus of thin film materials using self-deformed micromachined cantilevers. J. Micromech. Microeng. 1999;9:230-253. 
[19] Janssen G, M Abdalla, F Van Keulen, B Pujada, and B Van Venrooy. Celebrating the 100th anniversary of the Stoney equation for film stress: Developments from polycrystalline steel strips to single crystal silicon wafers. Thin Solid Films. 2009;517:1858-1867.

[20] Korsunsky AM, M Sebastiani, and E Bemporad. Residual stress evaluation at the micrometer scale: Analysis of thin coatings by FIB milling and digital image correlation. Surf. Coat. Tech. 2010;205:2393-2403.

[21] Guckel H, T Randazzo, and DW Burns. A simple technique for the determination of mechanical strain in thin films with applications to polysilicon. J. Appl. Mech. 1985;57:1671-1675.

[22] Guckel H, D Burnst, C Rutigliano, E Lovell, and B Choi. Diagnostic microstructures for the measurement of intrinsic strain in I thin films. J. Micromech. Microeng. 1992;2:86-95.

[23] He Q, Z Luo, and X Chen. Comparison of residual stress measurement in thin films using surface micromachining method. Thin Solid Films. 2008;516:5318-5323.

[24] Zhang T-Y, Y-J Su, C-F Qian, M-H Zhao, and L-Q Chen. Microbridge testing of silicon nitride thin films deposited on silicon wafers. Acta Mater. 2000;48:2843-2857.

[25] Zhou ZM, Y Zhou, CS Yang, JA Chen, GF Ding, W Ding, MJ Wang, and YM Zhang. The evaluation of Young's modulus and residual stress of nickel films by microbridge testings. Meas. Sci. Technol. 2004;15:2389-2394.

[26] Zhou Z, Y Zhou, M Wang, C Yang, Ja Chen, W Ding, X Gao, and T Zhang. Evaluation of Young's modulus and residual stress of NiFe film by microbridge testing. J. Mater. Sci. Technol. 2006;22:345-348.

[27] Herbert EG, WC Oliver, MP de Boer, and GM Pharr. Measuring the elastic modulus and residual stress of freestanding thin films using nanoindentation techniques. J. Mater. Res. 2011;24:2974-2985. 
[28] Zhang T-Y, X Wang, and B Huang. Microbridge testing of thin films. Mat. Sci. Eng. A. 2005;409:329-339.

[29] Nie M, Q-A Huang, and W Li. Measurement of material properties of individual layers for composite films by a pull-in method. J. Phys. Conf. Ser,. 2006;34:516-521.

[30] Saha R and WD Nix. Effects of the substrate on the determination of thin film mechanical properties by nanoindentation. Acta Mater. 2002;50:23-38.

[31] Krottenthaler M, C Schmid, J Schaufler, K Durst, and M Göken. A simple method for residual stress measurements in thin films by means of focused ion beam milling and digital image correlation. Surf. Coat. Tech. 2013;215:247-252.

[32] Sebastiani M, C Eberl, E Bemporad, and GM Pharr. Depth-resolved residual stress analysis of thin coatings by a new FIB-DIC method. Mat. Sci. Eng. A. 2011;528:7901-7908.

[33] Timoshenko S. On the correction factor for shear of the differential equation for transverse vibrations of prismatic bar. Philod. Mag. 1921;6:295.

[34] Volkert CA and ET Lilleodden. Size effects in the deformation of sub-micron Au columns. Philod. Mag. 2006;86:5567-5579.

[35] Cao Y, S Allameh, D Nankivil, S Sethiaraj, T Otiti, and W Soboyejo. Nanoindentation measurements of the mechanical properties of polycrystalline $\mathrm{Au}$ and $\mathrm{Ag}$ thin films on silicon substrates: Effects of grain size and film thickness. Mat. Sci. Eng. A. 2006;427:232-240.

[36] Arunasalam P, F Zhou, HD Ackler, and BG Sammakia. Thermo-Mechanical Analysis of ThruSilicon-Via Based High Density Compliant Interconnect. 2007;1179-1185.

[37] Ghidelli M, S Gravier, J-J Blandin, J-P Raskin, F Lani, and T Pardoen. Size-dependent failure mechanisms in ZrNi thin metallic glass films. Scr. Mater. 2014;89:9-12.

[38] Somà A and A Ballestra. Residual stress measurement method in MEMS microbeams using frequency shift data. J. Micromech. Microeng. 2009;19:095023. 
[39] Sebastiani M, E Bemporad, G Melone, L Rizzi, A Korsunsky, E Zschech, S Ogawa, and PS Ho. A new methodology for in-situ residual stress measurement in MEMS structures. in AIP Conference Proceedings. 2010.

[40] Kennedy M, D Bahr, C Richards, and R Richards. Residual stress control to optimize PZT MEMS performance. in MRS Proceedings. 2002. Cambridge Univ Press. 